\title{
Research, analysis, and assessment of the Bulgarian population's preparedness to provide first aid
}

\author{
Andrian Georgiev, Silvia Tsanova-Savova \\ International Business School - Botevgrad, Bulgaria \\ Medical College "Yordanka Filaretova", \\ Medical University - Sofia
}

\begin{abstract}
:
In Bulgaria, there are no statistics on the attitude of the society to the topic of first aid and the extent and scale of the trained people. This fact gave rise to the need to prepare and conduct a study, the results of which will illustrate more about the topic. The aim of this study is to understand more on whether people are ready to provide first aid and what makes them feel confident/insecure to help in case of an accident or acute illness. The methods used are developing and testing a questionnaire, collecting qualitative and quantitative data through an online survey and evaluation, analysis, and interpretation of the collected data. The results show, that a very small part of the respondents has sustainable knowledge and skills in first aid, which leads to a reduced readiness to help the casualty. This is rooted not in the unwillingness to help, but in the low confidence in the training received. The lack of regular repetitive training increases people's feelings of insecurity, prevents them from acting when needed, leading to a number of large-scale complications such as increasing the burden on health care, in the form of additional costs for subsequent hospital stays/treatment. The data collected from the study will be used to develop suggestions for the implementation of innovative training programs to fill the gaps in preparing the population for first aid, and subsequently on the social and health well-being of society by increasing survival rate and respectively saving lives.
\end{abstract}

Keywords: first aid, research, society, training, preparedness

\section{Introduction}

The need to acquire first aid skills follows from the objective physiology of the human body and its limits, which impose the need for rapid and urgent measures from the first person who arrived at the scene of the accident. After the third minute, without the presence of oxygen in the brain, irreversible processes begin to occur. The importance of first aid skills is also due to the fact that even the fastest ambulances in the world cannot arrive at the scene of an accident within less than 5 to 8 minutes. According to statistics provided by the UK National Health Service (UK NHS, 2017), the average time for emergency service (EMS) to arrive at the scene of a life-threatening accident requiring cardiopulmonary resuscitation (CPR) cannot be reduced to less than 7 minutes. Many people believe that 5-8 minutes until the ambulance arrives, is not that long time to keep a person alive, but Figure 1 shows an approximate timeline of an accident in which a casualty receives the first medical intervention and assistance, only after 15 minutes from the occurrence of the incident: 


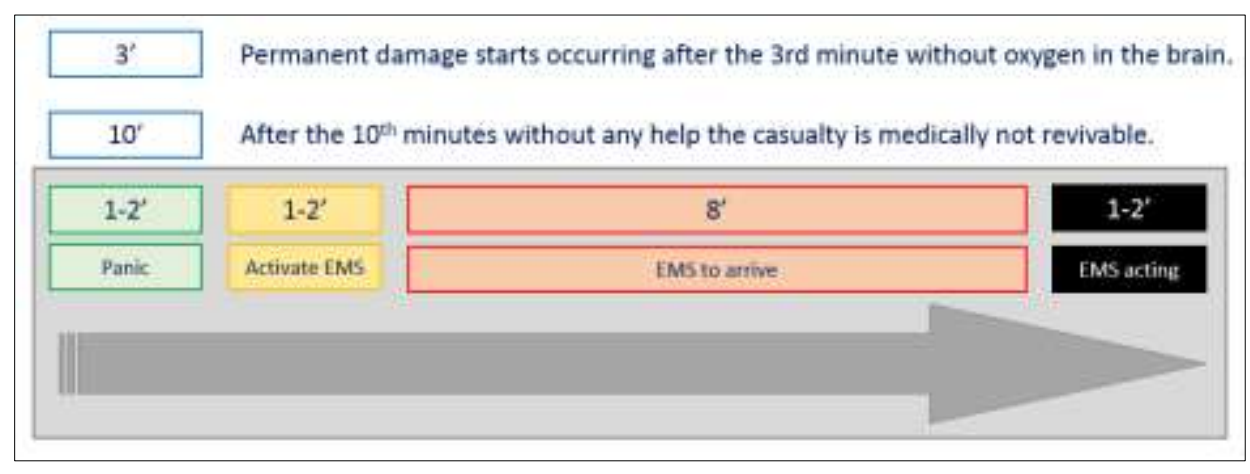

Figure 1. Accident timeline

The second example concerns one of the most life-threatening conditions - SCA. With it, the need for immediate action by eyewitnesses to the incident becomes even more important. According to European data (Gräsner J, 2016) (ERC, 2017) cases of SCA in Europe are 350,000 per year (compared to 475,000 cases per year in America (American Heart Association, AHA)). Approximately 100,000 of these cases could be saved if the cardiopulmonary resuscitation (CPR) turned out to be timely. $45 \%$ of SCA casualties survive only because an eyewitness or passer-by intervened and provided the casualty with immediate CPR.

The above data itself demonstrates that how important is that the population is well trained to promptly react in the case of an accident. But since in Bulgaria reliable statistics on first aid preparedness is hardly findable, that brings up the need for a study that will help to learn more on the topic.

This itself defines the aim of the study - to understand more on how prepared the Bulgarian population is in the matter of providing first aid in the case of an accident or acute illness. The study aim would be achieved by developing and conducting an online survey.

\section{Study SOCIETY AND FIRST AID \\ 1) Background and context}

In today's world, the need for first aid skills is increasingly urgent. Accidents happen often and the likelihood of witnessing one increases significantly. The timely and correct response in such situations, by witnesses or those who arrived first at the scene of the accident, in a very large percentage of cases can be lifesaving. That is why an increasing part of the population needs to be trained to provide first aid in the event of an accident or acute illness.

\section{2) Literature review of existing studies}

Previous literature review reports that national studies on first aid demonstrate the need of educating society on how to recognize the most common injuries and to start acting immediately after this. For example, a Norwegian study (Bakke H, 2016) on first aid in the case of out-of-hospital cardiac arrest (OHCA) from 2016, indeed shows that the state developed quite stable organizations to train the population. On the other hand, the survey results also evidence for the fact that the theoretical knowledge on how to proceed in case of OHCA or trauma is worse than expected. Another survey, in Germany (Schiefer J.L., 2017), on the level of knowledge to provide basic life support (BLS) was initiated due to the lack of understanding of how well the people are prepared to provide CPR in the case of OHCA. Previous data states that only $16.1 \%$ of the cases of OHCA received CPR by bystanders in Germany. This amount is quite low compared to other European countries (such as the Netherlands and Sweden) where this percent is above $60 \%$.

These examples provoke to learn more about what the current situation in Bulgaria is about first aid preparedness. As there is a lack of an adequate and modern statistic in the country in regard to the attitude of the society to the topic of first aid, a national online study was initiated in 2019 to learn more on the subject. The results of it would also contribute to bringing more insights on the degree and scale of the people trained in the modern guidelines for first aid. 


\section{3) Aim of the study}

The mentioned so far defines and the main aim of the study - to examine and identify the attitude and preparedness of the Bulgarian society to the first aid. In a narrow context, this includes studying several aspects such as the willingness of bystanders and/or witnesses of the accident to assist and provide help, general first aid skills, knowledge on how to provide high-quality CPR, willingness to use an automated external defibrillator (AED) in the case of SCA and 3 case study questions to check what is the theoretical preparedness of the studied object - Bulgarian society. Once the results are populated and the general findings are ascertained, they would be a base for proposing particular suggestions on how the general population's preparedness to provide first could be improved. Remark: the proposition for improvements is not a subject of the current study. The current study aims at only collecting data.

\section{4) Research questions}

Considering that the literature review finds out a very little data on Bulgaria population preparedness, this raises the following research questions:

1. Does the population of Bulgaria feel prepared to carry out CPR in case of SCA?

2. Does society accept the idea of placing AED in public places?

3. Is the public confident in the implementation of measures for the provision of first aid in case of an accident (reporting of readiness for action)?

4. Would the public's response to a life-threatening incident be adequate?

Answering these questions and using the outcomes of this national online study on first aid preparedness would benefit the organization which organizes and conducts first aid training courses. Knowing more about the topic and the general population's own assessment on first aid knowledge and skills will help these organizations (such as Red Cross and other NGOs/B2C) in what directions to add efforts in order to increase on the one hand the knowledge and skills for first aid and on the other hand, the willingness of bystanders to act in the case of life-threatening incidents and conditions.

\section{5) Research approach}

Like much of the research, this one begins with identifying and defining the problem that will be addressed. Once it has been determined, the object and subject of the study should be formulated (Mileva S, 2019). The research approach of the specific study follows a logical sequence and is organized in a vertical direction. It starts from ascertaining the global variable (problem), goes through determining the essence of the desired object of study, and reaches its specific characteristics and specifics - subject. The development considers more than one of the subjects (characteristics) of the studied object - society. The figure below illustrates the transfer of this theoretical model (problem - object - subject) into specific dimensions and specifics of the present study (Figure 2).

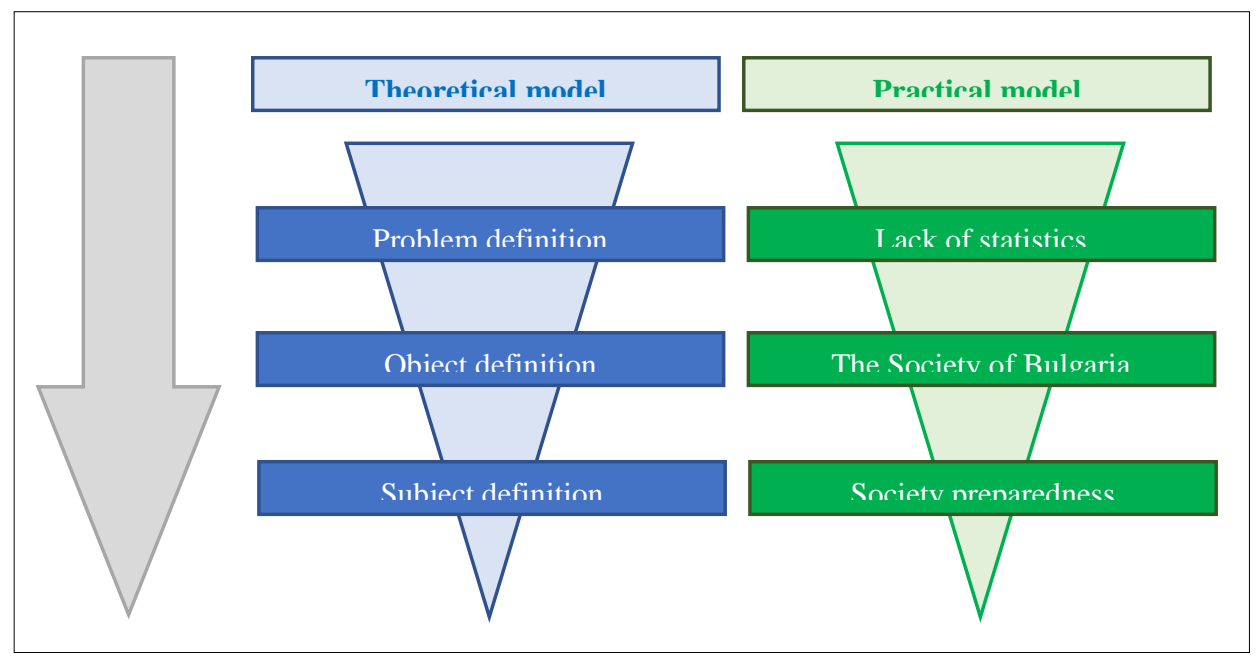

Figure 2. Model of determining the "problem - object - subject" of research 
Given the nature of the chosen method (online survey), the object of the study are people of all ages who can and do use the Internet freely.

\section{6) Methods and methodology}

The empirical nature of the study defines the survey methods used to achieve the set - developing, testing, and launching of a semi-structured online questionnaire/survey, including a good mix of quantitative (closed-ended questions), qualitative (open-ended questions), and informative pages (Mileva S, 2019). The mix of qualitative and quantitative questions is preferred as that approach allows the researcher to receive a broader perspective of the studies area, and at the same time to answer the already pre-defined research questions. Using the closed-ended question helps to collect data that would not need any interpretation, such as age, gender, location, etc., and respectively it is much easier for processing. On the other hand, applying open-ended questions contributes in a way where the researcher might receive a perception by the respondents for which s/he hasn't considered before starting the survey. Once all the desired data is collected, the information is synthesized and analyzed using a software solution for data processing.

As the survey is conducted through an open-access platform, a specific profile of the surveyed sample cannot be prepared. The aim is to collect as much data as possible for a specific period of time. This, itself, defines the preferred sample type - all the people who respond to the survey, have internet access and can use it freely. Additionally, using this approach is cost-effective, as the survey is self-funded. Although that the chosen sample approach does not provide representativeness of the data, this serves well for the explanatory aim the study is created for. On the other hand, this approach allows achieving the study's ultimate goal, i.e. to understand more on the topic for the first aid preparedness of the society and to fill the gap of the adequate statistic on the topic in the framework of Bulgarian context.

A representative limitation of the selected approach (online survey) is to achieve gender balance of the selected sample. Based on the online platform statistics (Figure 3), the most active profile seems to be educated women, 25-44 years old. To eliminate that limitation, for future similar studies, the gender balance could be equalized by using offline/F2F interviews and selectively approaching and interviewing males.

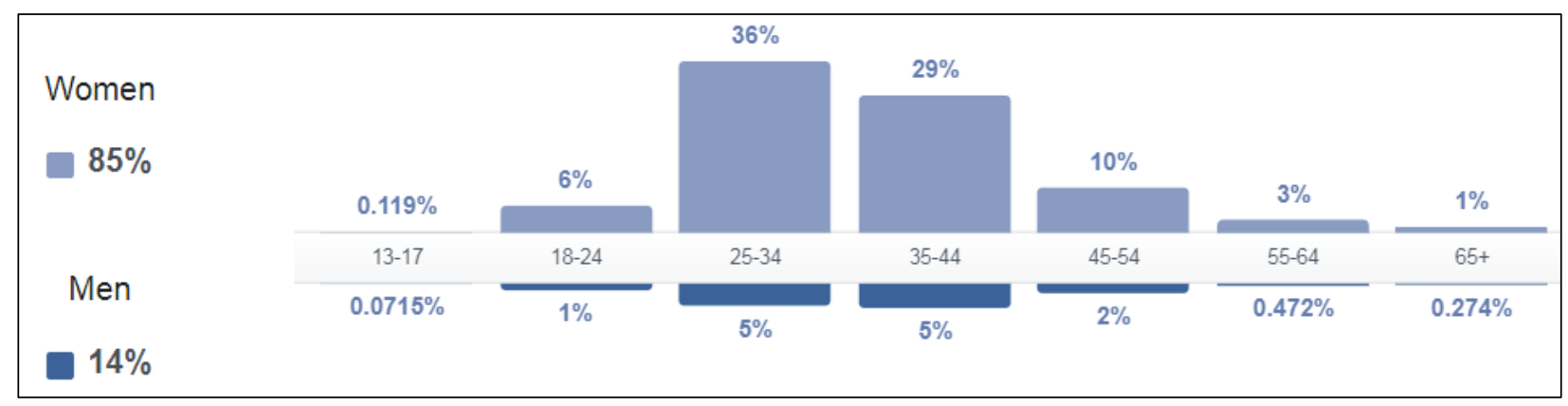

Figure 3. Presence of different age groups in the online space

Source: Facebook page of FirstAidbg.com First Aid School, based on 25,300 users.

\section{7) Survey scope}

The survey duration was approximately 8 months (February - October 2019). During the period, while the study was on the field, it was able to collect 3334 completes/completed interviews from all over the country. The survey covered all 28 districts of Bulgaria, and the initial goal of reaching a minimum of 15 people per district was met. $94.8 \%$ of the completes/respondents live in Bulgaria. Most participants in the study are women aged 25 to 44 who live in Sofia, Bulgaria, and have a university degree, bachelor's, or master's degree.

The survey has been composed and organized into 5 different categories. Before moving ahead to the actual questions and with the introduction of the GDPR in 2018, the survey first asks the respondents for their consent to participate in the research process. The first section, Demographic, was asking the respondents 
about their gender, age, location (Bulgaria [district] / abroad), educational level. These demographic characteristics were needed in order for the research process to make sure that it covers the entire country. Then the following category was related to the knowledge of how to provide high-quality CPR. Except for its research nature, the survey also had the idea to educate the people who take it. For example, the first question of this section was asking: "Do you know what cardiopulmonary resuscitation is?". In case that the respondent selects an answer "No", on the following screen would be presented the definition of the term CPR. The CPR section was examining whether the respondent has gone through CPR course, when was the last time this was done, what was the duration of the course, whether the respondent trust that the society should go through mandatory CPR training sessions if the respondent would provide CPR to a stranger and if not - why, etc. The third section was related to the knowledge and usage of an AED. Once again, the same educational approach was used. The respondents were asked if they know what AED is and if they mentioned "No", on the next screen they are able to read more about what AED is and how it operates. The fourth section is related to the general first aid, composed of questions, asking if the respondent know what first aid is, according to the ERC guidelines, if the respondent was in a situation where s/he needed to provide first aid, was s/he afraid to provide such, in case s/he didn't what was the main reason not to do so, whether the respondent went through a first aid course, how long ago, where, would s/he sign up for another course, if there is a first aid measure which s/he would not apply, why so if there is any, etc. The last section included 3 practical case study questions in order to reveal the theoretical preparedness of the society on how people should proceed in the case of life-threatening accidents. Last but not least, the survey also asked the respondent if they are going to sign up for a first aid course and in what circumstances.

\section{Results and discussion}

Section 1: Demographics

The first section, Demographics was concluded that the period of conducting the national online survey for the preparation of the population in Bulgaria for first aid is February-October 2019 (8 months). The total number of respondents is 3334, of which 756 are men and 2578 are women. The survey covered all 28 districts of Bulgaria, and the initial goal of reaching a minimum of 15 people per district was met. The majority of participants in the study are women aged 25 to 44 who live in Sofia, Bulgaria, and have a university degree - bachelor or master. This was the expected profile of the respondents, given the online presence of different age groups and participants grouped by gender according to Facebook Insights.

\section{Section 2: CPR}

The results of the study in its second section, namely $\boldsymbol{C P R}$, show that almost half of the participants underwent CPR training. Of these, only 1/5 have done so in the last 1 year. These are only 334 people from the entire sample or only about $10 \%$ of it. It is clear that in this area Bulgaria is still far from the required European minimum of $30 \%$ trained by the population.

On the other hand, over $98 \%$ of the participants believe that the population should undergo training on the CPR and would spend between 4 hours and 2 days every 2-3 years to train. Here the recommendation is to devise specific incentive programs to motivate the population to undergo such training and to do so with greater frequency.

The results of the survey clearly show that more than $86 \%$ of participants would perform CPR on a stranger if necessary, with most knowing that delaying CPR for a person with a stopped heart can lead to permanent brain damage. Those who would not undertake a stranger's CPR are most often concerned about their lack of training, which is another addition to the need for adequate training programs.

\section{Section 3: AED}

When analyzing the $\boldsymbol{A E D}$ section, the data from the obtained results exceed the pre-set expectations. Nearly $92 \%$ of the participants believe that placing an AED in public places is important and this gives grounds for a serious impetus and initiation of discussions on the topic at the national level. This is compounded by the fact that more than half know what an AED is and more than two-thirds of them would use an AED if needed without worrying about it being a machine that produces a controlled electrical impulse. One of the 
most important things that people need and realize in the AED debate is that they can't hurt a victim by using the machine. It cannot be "forced" to produce an electrical impulse unless it deems it necessary.

\section{Section 4: First Aid}

In the fourth section of the survey, First aid, the results show that more than $1 / 3$ of the respondents found themselves in a situation where first aid had to be provided and $76 \%$ of them did so, driven by the desire to help. Those who did not intervene most often felt insecure about their skills and remained passive while others acted. This fact, once again, supports the recommendation for regular refresher courses on first aid.

Unfortunately, however, most people have received first aid training only once in their lives and only in preparation for prospective drivers. According to modern organizations that issue guidelines for first aid by a layman, this is not enough, and such refresher courses must be taken at least once a year. In contrast, $70 \%$ of these people believe that this one course has prepared them for action in the situation in which they find themselves.

Most of those who believe that first aid courses have not adequately prepared them for the emergency situation in which they find themselves, say that this was most often due to little practical exercise and lack of refresher knowledge, which proves that because first aid techniques are practical and must be practiced regularly.

CPR, AED usage, severe bleeding control, which are life-saving measures, and the survival of the casualty depends on these, appears to be the most concerning for the respondents. Among the listed measures that people would not apply, there are exclusively medical manipulations that are not applied by non-medical persons when providing first aid.

\section{Section 5: Additional questions}

The answers to the additional questions (last section) on specific situations for first aid show that the majority of the respondents know the basic and modern algorithms for first aid. Combined with answers from the section on measures they would not implement, they clearly demonstrate the need to refresh and consolidate knowledge to gain confidence in a critical situation, which is part of the recommendations based on the results of the study.

The data show that almost half of the participants would enroll in a first aid course, most often if it focuses on daily risks, family, and children and is held nearby, outside working hours, and is free or paid by the employer.

The aim of the study was to gather enough information to prepare the population of Bulgaria for first aid. Other scientific papers aim to qualitatively prove or disprove a preconceived hypothesis. Due to the lack of any adequate statistics concerning Bulgaria, this study aimed to accumulate quantitative information. As a result of the results of this study, trends were identified and future guidelines for building first aid training programs were presented.

The sub-goal of the research was also achieved, namely, to study the attitude and readiness of the society to accept the idea of placing an AED in public places. Once a positive attitude has been established, this gives the green light to initiate discussions at the national level to place AED in public places.

The quantitative goal of the study was also achieved - it reached 3334 people, which is far above the expectations of "at least 1000 people" and managed to take a snapshot of society and its attitude to first aid. The survey covered all 28 districts of Bulgaria with at least 15 participants from a district and in accordance with the Law on Large Numbers, might be considered as representative.

\section{Conclusion}

1) Recommendations based on the results obtained and analyzed from the national online survey 
Based on the results obtained, the following recommendations can be made about the development of programs for periodic training / first aid courses. These programs should be developed by professionals involved in such training. Although the BRC specializes in first aid courses to obtain a driving license, they also appeal and encourage similar programs to be developed (Mediapool, 2019).

It seems that the driving license course is the only mandatory spot where a person needs to go through a first aid class (for reference, it is a local law that in Bulgaria, in order to receive your driving license, all the candidates should go through a mandatory Red Cross first aid training). At that point in time, this is necessary to be done once and if a person has a driving license for 10 or 15 years, this would mean that the last time s/he went through a first aid course was 10-15 years ago. In that light, society should go through first aid refreshes. As a good start, the first aid refreshing courses could relate only to the life-threatening conditions (choking, severe bleeding, unconscious person, SCA). Cases, where if the bystander or the witnesses of the accident does not start acting immediately, the casualty would start dying after the 3rd minute. Having in mind that organizations such as ERC, AHA, IFRC recommend first aid training to be passed once per year, a pilot program could be developed to test how this would be accepted by the society in order to measure how often these courses should be repeated. These first aid refreshers would be enough if they are practical and the duration is 3 academic hours. That would help eliminate, or at least to reduce, the society's concerns to provide first aid in case of an accident and will significantly increase the willingness of bystanders to assist in case of need.

Other questions here raise: "What if a person prefers not to be a driver?", "How these people would be required to go through first aid training?", "Where they would go through such training - in local communities, medical and/or recreation centers, specialized course?". All these research questions could be included in the guidelines for another study on the topic in order to cover a broader amount of population.

Given the number of victims, as a result of SCA, to organize and conduct specialized courses on CPR and AED. Such courses (Basic Life Support) have been developed by ERC. The First Three Minutes Foundation is the only organization in Bulgaria that is authorized to conduct them on ERC's behalf. Initiation of activities for a change of the legal base in Bulgaria and regulation of the use of AED by non-medical persons; placing AED in public places, starting in Sofia as a pilot project, and continuing to subsequent major cities (Manusheva M, 2020). Nowadays, there is such a municipality program in Plovdiv that installed already 8 AEDs for public usage. In Sofia, there are 2 AEDs for public usage.

The concrete plan for such first aid training programs would be a subject of another study, which will collect data after a certain period of time. Based on the results and comparisons between stage 1 (the current study) and stage 2 (future), measuring the gaps and weak points, a particular agenda would be proposed. That plan would include who has to organize such courses, where they should be conducted, what should be the course duration, how often these courses should be repeated, what would be the testing/pilot groups for these courses, etc. Introducing such programs would be a time-consuming procedure, as it would require multidisciplinary work among institutions (such as the Ministry of Education, the Ministry of Health, the Ministry of Interior, etc.) and organizations from NGO (or B2C) sector which will appear as course providers.

\section{2) Guidelines for future research on the topic}

Guidelines for future research on the topic of first aid are to conduct a study after 2.5-3 years (mid2021/2022) to consider the change in preparation (deterioration, maintaining the same level, increase) and attitudes to first aid (negative, neutral, positive) from society. Such statistics are needed so that training organizations have a clear vision of where they should go. Given the fact that the state cannot create a unified system to train the entire population, society is just beginning to look for ways and alternatives for self-education on the subject. For this reason, the estimates for increasing the capacity of the society are optimistic, but in order to really take this into account, it is recommended that a similar (or the same) study be conducted after 2-3 years. 
Until then, we can draw on the experience of countries such as Norway, Germany, and Austria, which according to IFRC's report "First aid for a safer future Focus on Europe - Advocacy report 2009", published in 2009, are countries with over $80 \%$ of the population trained in first aid (IFRC, 2009). A detailed overview of the experience of these countries can be found in the article "The experience of the leading European countries in preparing the population in case of an accident", published in the collection Tourism and global crises, Veliko Tarnovo University, I\&B Publishing House, 2021 (Georgiev A, 2021).

\section{Reference:}

1. American Heart Association. CPR Facts and Stats. How CPR is changing (and saving lives). https://tinyurl.com/y8y279ne

2. Bakke, H., Steinvik, T., Angell, J., Wisborg, T. (2016). BMC Emergency Medicine. A nationwide survey of first aid training and encounters in Norway. https://tinyurl.com/ya3b3c3m

3. European Resuscitation Council (2017). European Registry of Cardiac Arrest - Study TWO (EuReCa TWO). https://www.eureca-two.eu/

4. Georgiev, A (2021). The experience of the leading European countries in preparing the population in case of an accident. Tourism and global crises. Veliko Tarnovo University, I\&B Publishing house. 2021. p. 830

5. Gräsner, J., Lefering, R., Koster, R., Masterson, S. et al. (2016). European Resuscitation Council. EuReCa ONE - 27 Nations. ONE Europe. ONE RegistryA prospective one month analysis of out-ofhospital cardiac arrestoutcomes in 27 countries in Europe. https://pubmed.ncbi.nlm.nih.gov/27321577/

6. IFRC (2009). First aid for a safer future Focus on Europe - Advocacy Report. Health and care department 2009

7. Manusheva, M (2020). Sofia is considering installing defibrillators in public places. OFFFNews. 2020. https://tinyurl.com/y72cdtlc

8. Mediapool (2019). Proposal for periodic repetition of the Bulgarian Red Cross driver's course. https://tinyurl.com/ybz35f66

9. Mileva, S (2019). Handbook of the discipline Marketing Research. Botevgrad, IBS, 2019, Topic 6. Algorithm for conducting marketing research, p. 16; Topic 8. Qualitative methods for collecting primary information, p. 22; Topic 9. Quantitative methods for collecting primary information, p. 25

10. National Health Services England (2017). Ambulance response time. https://tinyurl.com/yclbys21

11. Schiefer, J.L., Schuller, H., Fuchs, P.C., Bagheri, M., Grigutsch, D., Klein, M., et al. (2020). Basic life support knowledge in Germany and the influences of demographic factors. PLoS ONE. https://doi.org/10.1371/journal.pone.0237751 